\title{
Acute myocarditis following varicella zoster infection in an immunocompetent adolescent: An uncommon complication
}

\author{
Emine Azak, M.D. ${ }^{a}$ and Ibrahim I. Cetin, M.D. ${ }^{a}$
}

\begin{abstract}
Myocarditis is a serious complication of varicella zoster virus infection. A 15 year-old boy was admitted to the Emergency Department for chest pain, tachycardia and hypotension. An electrocardiogram showed sinus tachyicardia. Cardiac biomarkers were elevated and echocardiography revealed left ventricular apical, inferolateral, septal hypokinesis, and mitral regurgitation. Varicella zoster virus serum immunoglobulin $\mathrm{M}$ antibody was positive. The patient was discharged without any sequelae.

Key words: adolescent, myocarditis, varicella zoster virus.
\end{abstract}

http: / / dx.doi.org/10.5546/ aap.2020.eng.e284

To cite: Azak E, Cetin II. Acute myocarditis following varicella zoster infection in an immunocompetent adolescent: An uncommon complication. Arch Argent Pediatr 2020;118(3):e284-e287.

\section{INTRODUCTION}

Acute myocarditis is a major cause of acquired heart disease worldwide in pediatric population. Various viruses such as varicella zoster virus have been reported in the etiology. However, since administration of vaccine worldwide, reports of myocarditis caused by varicella zoster virus have become sporadic. ${ }^{1-5}$ To our best knowledge only a few cases of myopericarditis caused by varicella zoster virus have been reported, most frequently in children, seldom in immunocompetent adults. We report on a case of a myocarditis caused by varicella zoster virus in a previously healthy adolescent, with a typical development and a fast and complete recovery.

a. Department of Pediatric Cardiology, University of Health Sciences, Ankara Child Health and Diseases Hematology Oncology Training and Research Hospital, Ziraat Mah, Ankara, Turkey.

E-mail address:

Emine Azak, M.D.: azakemi@gmail.com

Funding: None.

Conflict of interest: None.

Received: 3-8-2019

Accepted: 10-28-2019

\section{CASE PRESENTATION}

A 15-year-old male previously healthy patient was admitted to our hospital with severe chest pain, palpitation and fever. He had no medical history of serious previous illnesses or family history of cardiovascular disease. Five days before the admission, the patient began to be subfebrile with cough, dyspnea and chest pain. There was a history of contact with a child who had chickenpox a few weeks ago. The patient was previously vaccinated for chickenpox. In his physical examination, there were a few dry vesiculopapular rashes on trunk, with no audible murmurs or pericardial friction rub. The blood pressure was 90/60 $\mathrm{mmHg}$ and body temperature was $37.7^{\circ} \mathrm{C}$. An electrocardiogram (ECG) showed sinus tachycardia with no voltage suppression and pathologic ST segment elevation or T inversion (Figure 1). Laboratory evaluation revealed a total leukocyte count of $9.5 \times 10^{9} / \mathrm{L}$, erythrocyte sedimentation rate was $2 \mathrm{~mm} / \mathrm{hr}$, C-reactive protein was $15 \mathrm{mg} / \mathrm{L}$ (normal range, 0.8-3.1 mg/L). Creatine kinase-MB (CK-MB) was elevated to 36 (0-24 U/L) and cardiac troponin-I (cTnI) was elevated to $5.94(0-0.04 \mathrm{mcg} / \mathrm{L})$. B-type natriuretic peptide (BNP) levels were also elevated from $266 \mathrm{pg} / \mathrm{ml}$ to $469 \mathrm{pg} / \mathrm{ml}$ $(0-70 \mathrm{pg} / \mathrm{ml})$ after 12 hours of admission. The levels of BNP and cTnI during the hospitalization are shown in Figure 2. The complete blood count, serum albumin, creatinine, urea, and hepatic transaminase activities, C-reactive protein and erythrocyte sedimentation rate were within normal ranges. An enzyme-linked immunosorbent assay (ELİSA) was positive for varicella zoster virus with elevated immunoglobulin $\mathrm{M}$ and immunoglobulin $\mathrm{G}$ levels [30.5 index (> 11 index) and > $53.6 \mathrm{ng} / \mathrm{mL}$ (5-9 IU/ $\mathrm{mL})$, respectively]. Coxsackie, echo, influenza $A$ and B, cytomegalovirus, and Esptein Barr viral titres were all negative. Decreased left ventricular contractility (fractional shortening $27 \%$, ejection fraction $53 \%$ ), mild mitral regurgitation, dilated left ventricle, and apical, inferolateral and inferoseptal hypokinesis were found on the echocardiographic examination. The patient was admitted to the pediatric intensive care unit and 
was started on non-steroidal anti-inflamatory drug (ibuprofen, $15 \mathrm{mg} / \mathrm{kg} /$ day), angiotensin converting enzyme inhibitor (enalapril, $0.1 \mathrm{mg} /$ $\mathrm{kg} /$ day) and intravenosus immunoglobulin (IVIG, $1 \mathrm{~g} / \mathrm{kg}$ per day for 2 days). The CK-MB, cTnI and BNP levels gradually returned to normal (18 U/L, $0.01 \mathrm{mcg} / \mathrm{L}$ and $65 \mathrm{pg} / \mathrm{ml}$, respectively) and a new echocardiography showed normal cardiac function (fractional shortening $38 \%$, ejection fraction $68 \%$ ). After 10 total days of hospitalization, the patient was discharged with instructions to take ibuprofen for 10 days and enalapril for 3 month and to limit his physical activity for 4 to 6 weeks. In addition, varicella zoster virus immunoglobulin M level ( 0.17 index) and immunoglobulin $\mathrm{G}$ levels were significantly elevated $(96.4 \mathrm{IU} / \mathrm{ml})$ at 4 weeks of follow-up.

\section{DISCUSSION}

Acute myocarditis is a major cause of acquired heart disease in pediatric patients. Various viruses have been reported in the etiology. Diagnosis of myocarditis or myopericarditis is rare during varicella -primary infection of VZV and sporadic

FIGURE 1. The electrocardiogram showing sinus tachycardia and normal ST-T segment on admission

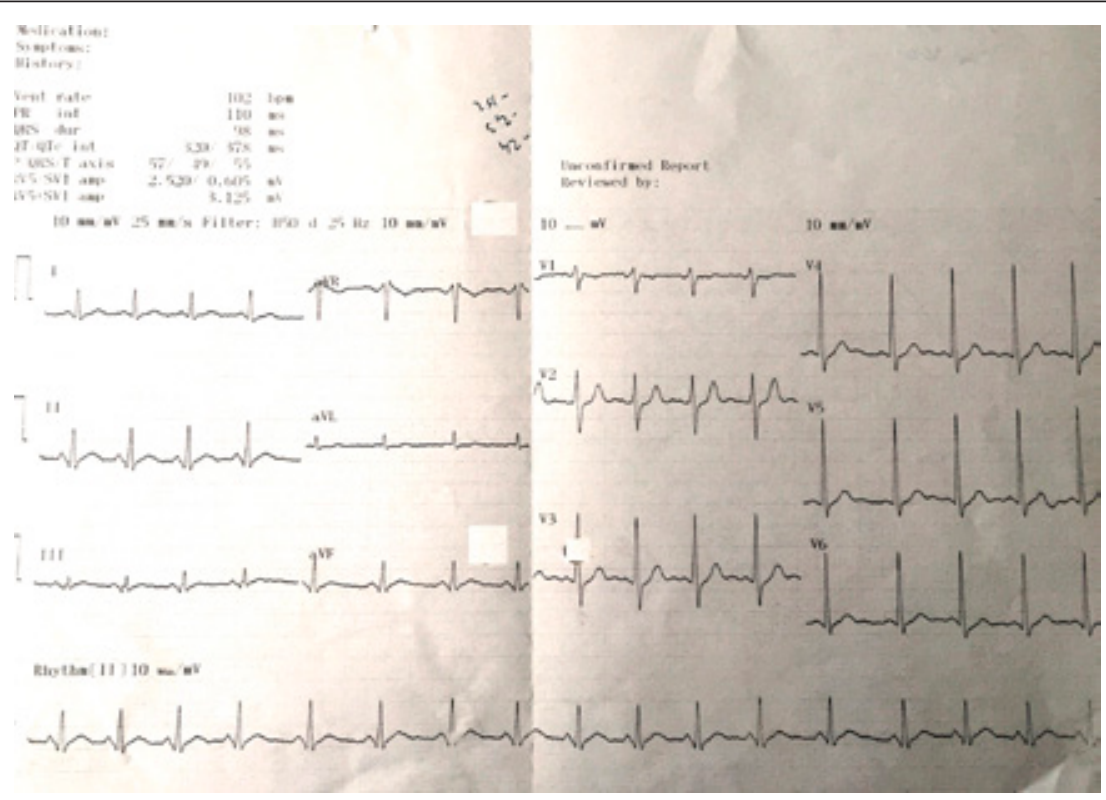

FIGURE 2. Troponin I and B-type natriuretic peptide levels during hospitalization

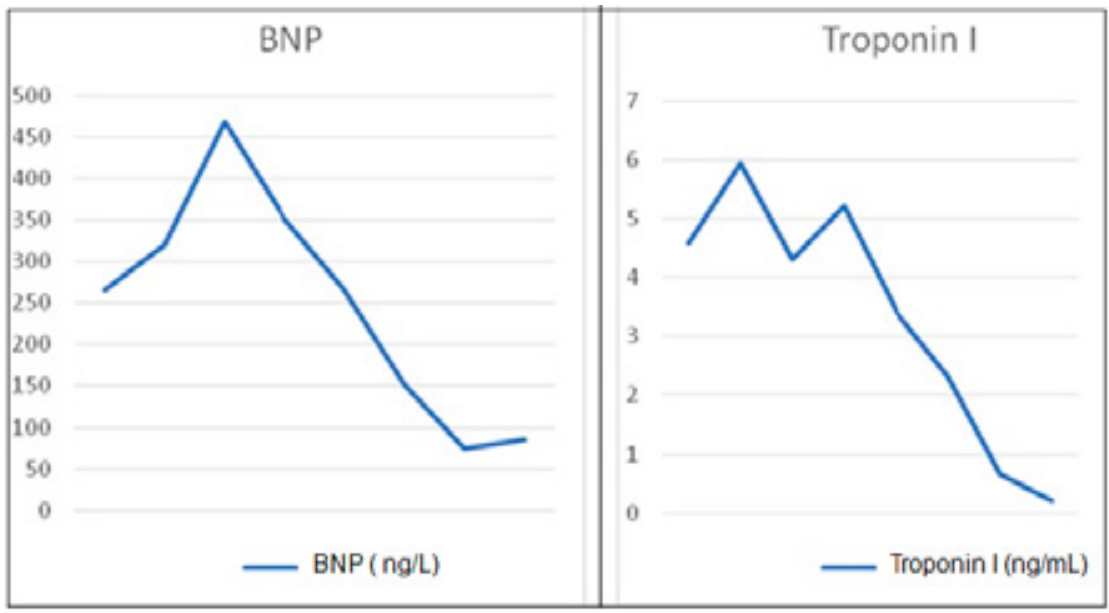

BNP: B-type natriuretic peptide. 
in zoster- reactivation of latent VZV. Only few such cases have been described, in which varicella zoster virus was first reported in 1953. ${ }^{1,6-8}$ However, with the global administration of the varicella vaccine, reports of myocarditis caused by varicella zoster virus have become sporadic. Varicella zoster virus infection is a common childhood infection and it is conceivable that subclinical infection or myocarditis may contribute to some cases of idiopathic dilated cardiomyopathy. ${ }^{5-8}$ Varicella zoster virus is one of the cardiotropic viruses. But, varicella is very rarely associated with cardiac sequelae. Cardiovascular complications of varicella zoster virus infection include endocarditis, myocarditis, pericarditis and arrhythmias. ${ }^{2-5}$ Cardiac sequels of varicella zoster virus infection are rare but can cause mortality and morbidity when it occurs. Since suspected varicella myocarditis in children may be associated with severe complications, including progressive heart failure as well as malignant arrhythmias and sudden death, the consensus is that these patients should receive medical intensive $\operatorname{care}^{7-11}$. Myocarditis associated with a viral illness is an inflammatory process that can lead to temporary or permanent damage to the myocardial structure and conduction system. The histopathological findings of varicella myocarditis are similar to other viral myocarditis. Clinical presentations of viral myocarditis can range from asymptomatic course to acute fulminant disease such as cardiovascular collapse. However, the majority of the cases is subclinical or found to have minor " $\mathrm{T}$ " wave changes on the ECG. $3-5,9$

Owing to the rarity of this disease, however, there are no generally accepted therapeutic guidelines for varicella myocarditis. The treatment of acute varicella zoster virus myocarditis is usually supportive but acute coronary syndrome should be excluded before administering treatment. Myopericarditis can mimic acute coronary syndrome, and the distinction can be subtle in an atypical clinical presentation. According to publications, in these cases, it is emphasized that performing coronary angiography is a safer and more prudent initial approach. After exclusion of coronary artery occlusion in these patients, management of myopericarditis includes inpatient treatment with nonsteroidal anti-inflammatory drugs, monitoring myocardial function, and, if necessary, correcting left ventricle dysfunction or arrhythmias. After the patient is discharged from the hospital, it is recommended to restrict physical activity for 4 to 6 weeks. ${ }^{2-5}$
Acyclovir is the antiviral agent that commonly used in the treatment of immunocompetent adults and children with varicella zoster virus and several reports suggest clinical benefits in adults with varicella zoster virus complications. However, because of the rarity of varicella zoster virus myocarditis there is no information to support its efficacy. ${ }^{4-8}$ Drighil et al., speculate that acyclovir may be beneficial in varicella zoster virus myocarditis if presentation occurs during the stage of viral replication in the myocardium. ${ }^{3}$ As is known, varicella zoster virus replication generally ends between the third and seventh day of the onset of vesicular rash, therefore, we did not administer acyclovir treatment because the patient only had dry vesicular rash. Laboratory tests are required to confirm varicella zoster virus infection, especially when a skin lesion is not typical or apparent. An ELISA test was positive for varicella zoster virus with elevated levels IgM and IgG. After varicella zoster virus infection, IgG and $\operatorname{IgM}$ antibodies appear 2 to 5 days after the rash and show the highest titers at 2 to 3 weeks. The varicella zoster virus IgM antibody levels then rapidly decrease and cannot be detected at 1 year after infection, and the IgG antibody levels gradually decrease, showing positive test results for several years. ${ }^{1,8,11,12}$ We suspected that acute viral myocarditis associated with varicella zoster virus due to the marked increase of varicella zoster virus Ig G levels 4 weeks after varicella zoster virus Ig M positivity. Diagnosis of myocarditis in this case was made based on echocardiogram, clinical history and on the clinical presentation of an acute varicella zoster virus infection, and was serologically confirmed by ELISA test. But, the use of acyclovir did not appear to be beneficial.

In conclusion, myocarditis is a serious complication of varicella zoster infection and heart failure may be fulminant. But, limited information is available related to myocarditis during varicella zoster virus infection. The development of tachycardia and chest pain in patients with varicella should signal the possibility of myocarditis. The clinical findings of varicella infection especially in adolescents may not be visible at the time of admission, so the physicians should be aware of potential cardiovascular complication of varicella infection. In atypical clinical situations, we believe that myocardial infarction and acute coronary syndrome should be excluded before proceeding with treatment. 
Cardiac biopsy is the gold-standard diagnostic test but is not usually performed in clinical practice..$^{9-11}$ Clinical suspicion supported by serology is sufficient for the diagnosis of varicella zoster myocarditis. There are no clear guidelines for the management of patients with myocarditis. Combination of acyclovir and IVIG has been reported to be life-saving in these patients. ${ }^{11-13}$ Acyclovir, an inhibitor of herpes virus DNA replication, was not used in the treatment of this child. Because of the rarity of varicella myocarditis, there is no specific data to support its effectiveness for this particular indication. But, acyclovir is of clear benefit in the treatment of immune-compromised patients with acute varicella.

With this report we want to point out that varicella zoster virus can cause myocarditis in adolescence, which must be taken into consideration in differential diagnosis, and that an early diagnosis and adequate therapy can help complete recovery.

\section{REFERENCES}

1. Abrams D, Derrick G, Penny DJ, Shinebourne EA, et al. Cardiac complications in children following infection with varicella zoster virus. Cardiol Young. 2001; 11(6):647-52.

2. Kao KL, Yeh SJ, Chen CC. Myopericarditis associated with varicella zoster virus infection. Pediatr Cardiol. 2010; 31(5):703-6.
3. Drighil A, Hammiri AE, Belmourida F. Chest Pain in a 17-Year-Old Girl with Chickenpox. Pediatr Ann. 2015; 44(9):359-65.

4. Elikowski W, MarszałekA, Malek-Elikowska M, GanowiczKaatz T, Mozer-lisewska I. Myopericarditis in a 23 year old male with herpes zoster. Pol Merkur Lekarski. 2016; 40(236):97-101.

5. De A, Myridakis D, Kerrigan M, Kiblawi F. Varicella myopericarditis mimicking myocardial infarction in a 17-year-old boy. Tex Heart Inst J. 2011; 38(3):288-90.

6. Donoiu I, Istratoaie O. Varicella-zoster myocarditis mimicking acute myocardial infarction. Curr Health Sci J. 2014; 40(1):78-80.

7. Waagner DC, Murphy TV. Varicella myocarditis. Pediatr Infect Dis J. 1990; 9(5):360-3.

8. O'Grady MJ, MoylettE. Cardiac-related varicella mortality in childhood: a literature review with clinical experience. Pediatr Cardiol. 2011; 32(8):1241-3.

9. Tsintsof A, Delprado WJ, Keog AM. Varicella zoster myocarditis progressing to cardiomyopathy and cardiac transplantation. Br Heart J. 1993; 70(1):93-5.

10. Alter P, Grimm W, Maisch B. Varicella myocarditis in an adult. Heart. 2001; 85(1):E2.

11. Kelly E, Cullen G, McGurk C. When an MI is not an MI: a case of varicella zoster myocarditis. Cardiology. 2008; 109(3):193-5.

12. Min SW, Kim YS, Nahm FS, Yoo da H, et al. The positive duration of varicella zoster immunoglobulin $\mathrm{M}$ antibody test in herpes zoster. Medicine (Baltimore). 2016;95(33):e4616.

13. Lu YC, Fan HC, Wang CC, Cheng SN. Concomitant use of acyclovir and intravenous immunoglobulin rescues an immunocompromised child with disseminated varicella caused multiple organ failure. J Pediatr Hematol Oncol. 2011; 33(8):e350-1. 